\title{
WILLIAM HOGARTHS ANGRIFF AUF DIE TAGESZEITENIKONOGRAPHIE
}

William Hogarths The Four Times of the Day (Abb. 1-4) von 1738 scheinen auf den ersten Blick mit der tradierten Tageszeitenikonographie, wie sie sich in einer Fülle von graphischen Serien seit dem 16. Jahrhundert niedergeschlagen hat, nichts zu tun zu haben. ${ }^{1}$ Auf den zweiten Blick entdeckt man zahlreiche subkutane Verweise auf die lange verbindliche Vorstellung von der Zeitenfolge. Doch damit stellt sich nicht etwa eine Rechtfertigung der Tradition in neuem Gewande ein, im Gegenteil. Offenbar kommt es zu einer Überprüfung, und das Resultat ist die Überzeugung von deren Inadäquatheit angesichts gegenwärtiger Verhältnisse, und Ausdruck findet dies, wie die Forschung durchaus feststellt, in einer Parodie klassischer Bildersprache. Ja, in vielerlei Hinsicht werden die Dinge geradezu auf den Kopf gestellt. Schon Reverend John Trusler in seinem »Hogarth Moralized« von 1768, das Hogarth zu einem reinen oder besser bloßen Moralisten macht und damit die Forschung bis in die Gegenwart auf unglückliche Weise bestimmt hat, attestiert Hogarth in dieser Folge eine Burleske auf die klassische Zeitenvorstellung. ${ }^{2}$ Im Übrigen sind beide Begriffe, Parodie und Burleske, letztlich nicht wirklich angemessen. Die Burleske betreibt den Spaß um des Spaßes willen, die Parodie behält die äußere Form bei und will den Inhalt als unpassend erweisen. Hier verhält es sich umgekehrt, und von daher wäre Travestie

1 Ronald Paulson: Hogarth's Graphic Works, 3. rev. Ed. London 1989, Kat.Nr. 146-149, S.103-108; Sean Shesgreen: Hogarth and the Times-of-the-Day Tradition. London 1983, der Arbeit wird im Folgenden einiges verdankt. 2 John Trusler: The Works of Mr. Hogarth Moralized. London 1768, 207217, zur Burleske ebd., 208. 


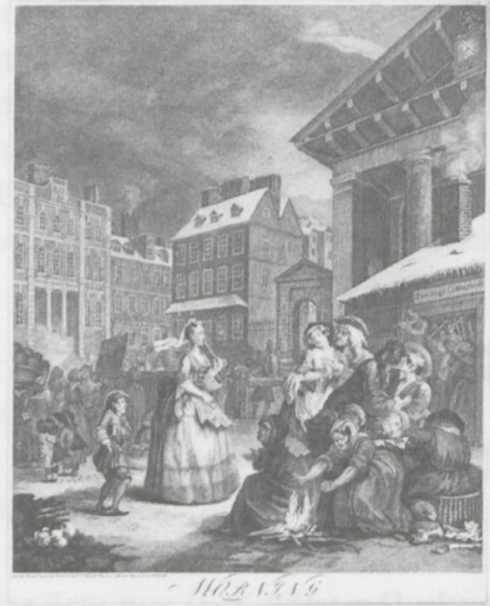

1 William Hogarth: Morning,

Radierung und Kupferstich, 1738, $49,0 \times 39,5 \mathrm{~cm}$

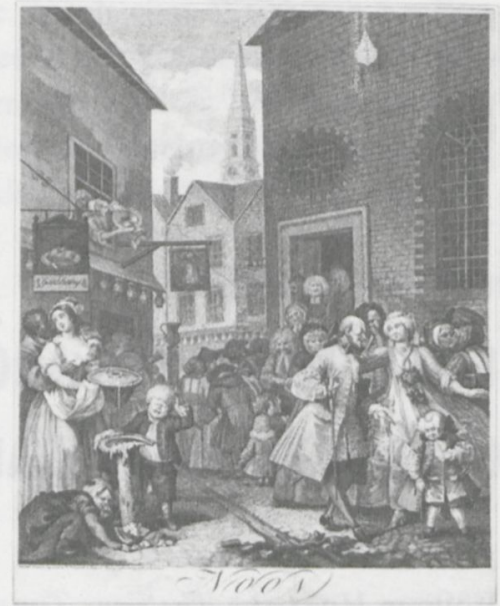

2 William Hogarth: Noon, Radierung und Kupferstich, 1738, $48,8 \times 40,2 \mathrm{~cm}$

die richtige Bezeichnung: Der Inhalt bleibt, aber er wird in einer lächerlichen Form präsentiert.

Zweierlei sei im Folgenden nicht verfolgt. Weder soll die Geschichte der quaternären Welt- und Zeitordnungsmodelle vorgeführt werden, die sich nicht nur bei den Jahreszeiten, Tageszeiten oder Lebenszeiten herausgebildet hat, sondern in einer Fülle anderer Bereiche. Sie gelten für die vier Winde, die vier Himmelrichtungen, lange Zeit für die Erdteile, für die vier Temperamente, die vier Paradiesströme, die vier Elemente, die vier Fakultäten oder - und das ist einer der Ausgangspunkte der genannten Modelle - für die vier Ovidschen Zeitalter der Welt, das goldene, das silberne, das bronzene und das eherne. Oft genug, um die Allgemeingültigkeit des Systems zu betonen, sind zudem mehrere der Modelle in den Darstellungen verschränkt, fast immer, um nur dies zu nennen, ist in eine Tageszeitenfolge die Vorstellung von den Jahreszeiten und Lebensaltern eingeschrieben. Noch soll die Herausbildung der speziellen Tageszeitenikonographie Thema sein, zumal ich dies andernorts verfolgt habe. ${ }^{3}$ Dazu müsste man auf den Zodiakus, auf die Monatsdarstellun-

3 Werner Busch: Von unvordenklichen bis zu unvorstellbaren Zeiten. Caspar David Friedrich und die Tradition der Jahreszeiten. In: Philipp Otto Runge - Caspar David Friedrich. Im Lauf der Zeit, hg. von Andreas Blühm, Ausst.-Kat. Van Gogh Museum Amsterdam. Zwolle 1996, 17-32. 


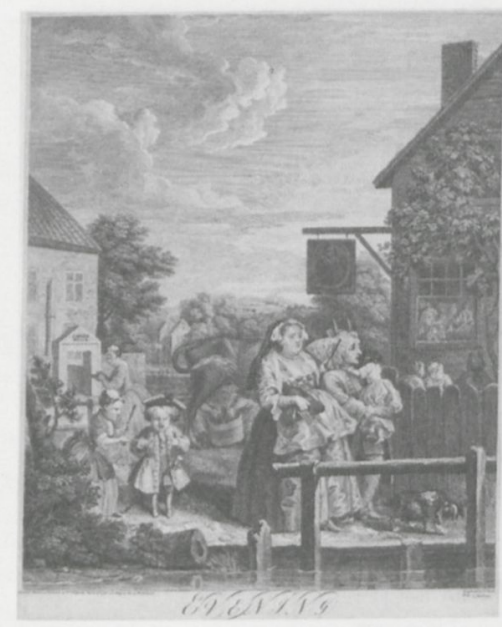

3 William Hogarth: Evening,

Radierung und Kupferstich, 1738, $48,6 \times 40,3 \mathrm{~cm}$

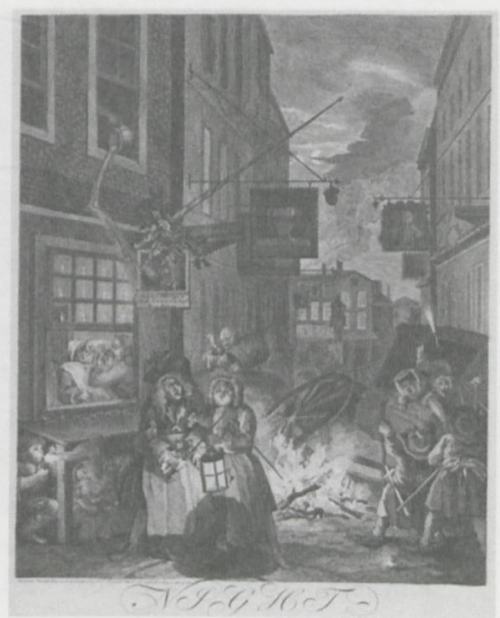

4 William Hogarth: Night, Radierung und Kupferstich, 1738 , $48,6 \times 40,2 \mathrm{~cm}$

gen in den Sockelzonen der Kathedralportale, auf die spätmittelalterliche Buchmalerei mit ihren Stundenbüchern rekurrieren, auf die Entwicklung der Jahreszeitenikonographie aus der Monatsabfolge etc. ${ }^{4}$ Es sei allein und auch das nur kurz - die entwickelte Tageszeitenikonographie, wie sie sich im späteren 16. Jahrhundert in den Niederlanden herausgebildet hat, vorgeführt, um eine Folie für das Folgende zu haben.

Als Beispiel sei Marten de Vos' Tageszeitenserie gewählt, von der man annimmt, dass sie um 1562 entworfen wurde. Sie ist um einiges später von Adrian Collaert gestochen worden. ${ }^{5}$ Im Stich werden die vier Blätter jeweils von einem lateinischen Vierzeiler begleitet, der auf die Moral von der Geschicht' abhebt, die Vergänglichkeit des Lebens betont und schon dadurch auf die Lebensalter verweist und den Gang der Dinge zumindest andeutungsweise unter das Gebot Gottes stellt. Insofern arbeitet die Serie bereits der barocken Zeitenvorstellung vor, die grundsätzlich sub specie aeternitatis zu denken ist und die, vor allem in Gedichtform bei Paul Fleming oder Gryphius, Erlösung und Ewigkeit beruft. ${ }^{6}$ Zudem weisen die Darstellungen zwei Register auf: oben in italianisierender Form eine göttliche Verkörperung in den Wolken,

4 Siehe dazu hier den Beitrag von Susanne Wittekind.

5 Shesgreen 1983 (wie Anm. 1), 26-36, Abb.1-4.

6 S. etwa die folgenden Gedichte, zitiert bei: Karl Otto Conrady: Das große 


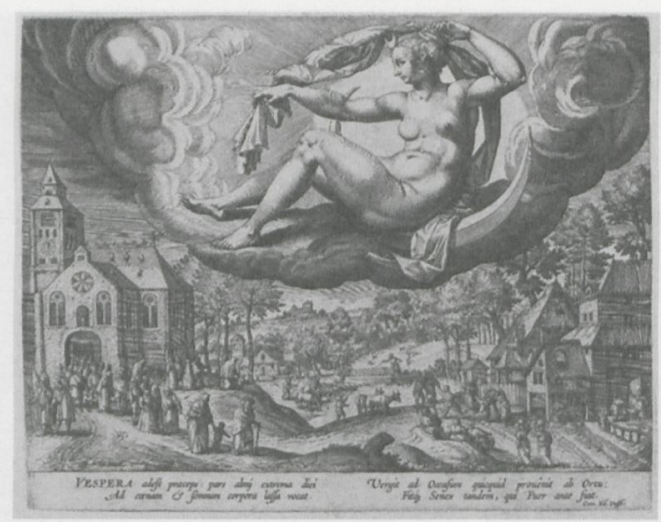

5 Adrian Collaert nach Marten de Vos: Mittag, Kupferstich, um $1600,20,8 \times 26,2 \mathrm{~cm}$

unten eine Landschaft mit vielfältigen Aktivitäten, deren Ikonographie nordischen Traditionen entspringt. Die Registerform hat ihren Ursprung in den Planetendarstellungen, es sei nur an den Hausbuchmeister erinnert. ${ }^{7}$ Im Laufe der Zeit entledigen sich auch in der niederländischen Tradition, und zwar bereits im 17. Jahrhundert, die Serien ihrer übergeordneten Götter, übrig bleibt die reine Landschaft mit tages- und jahreszeitentypischen Tätigkeiten.

Marten de Vos' Morgenszene wird von Aurora, der Morgenröte, beherrscht, sie ist jung und schön, blumen- und sternengeschmückt, wie es auch dem Frühling gebührt, die Landschaft zeigt erste Aktivitäten, die Herde wird auf die Weide getrieben, der Fischfang beginnt, die Kinder gehen zur Schule, dem Kleinkind wird das Essen bereitet. Den Mittag (Abb. 5) dominiert am Zenit der umstrahlte Sonnengott Apoll, er schaut auf das Erdengeschehen, zumindest auf den Teil, den auch die Verse loben. Auf der rechten Seite ist gezeigt, wie die Bauern fleißig gearbeitet, das Korn zu Garben zusammengelegt haben - was aus den jahreszeitlichen Aktivitäten der Monatsikonographie stammt. Jetzt erholen sich die Bauern im Schatten eines Baumes, essen und trinken, auch die Pferde haben Futter bekommen, einige Bauern arbeiten noch, das Feld

deutsche Gedichtbuch von 1500 bis zur Gegenwart, 2. Aufl. München und Zürich 1992, 31 (Fleming), 37-41 (Gryphius).

7 Vom Leben im späten Mittelalter. Der Hausbuchmeister oder Meister des Amsterdamer Kabinetts, Ausst.-Kat. Rijksmuseum Amsterdam; Städtische Galerie im Städelschen Kunstinstitut, Frankfurt a. M. Stuttgart - Bad Cannstatt 1985, Kat.-Nr. 117, Abb. S. 208-214, Taf. I und Abb. 1 und 2. 


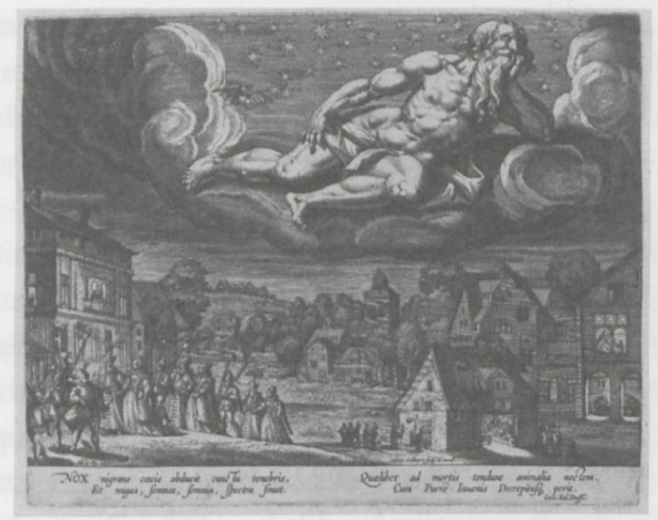

6 Adrian Collaert nach Marten de Vos: Nacht, Kupferstich, um $1600,20,8 \times 26,2 \mathrm{~cm}$

wird gepflügt, die Herde bewacht. Vom Land und der Landbevölkerung durch eine hohe Mauer getrennt, lässt es sich der Adel allzu gut gehen, Luxus und Nichtstun herrschen vor an reicher Tafel und beim amourösen Sichergehen im Garten, der Pfau daneben verweist auf die Gefahr des Hochmutes. Diese Trennung in richtiges und falsches Verhalten gilt es im Gedächtnis zu bewahren. Den Abend verkörpert Diana in ihrer Funktion als Mondgöttin. Auf dem Land wird die Herde wieder heimgetrieben. Der Vogelsteller hofft noch Beute zu machen. Ein Teil der Bevölkerung, vor allem in Gestalt der Älteren, ist bei der Abendvesper in der Kirche gewesen und nun auf dem Heimweg. Der andere Teil amüsiert sich nach des Tages Arbeit beim Tanz oder sitzt schon beim Abendbrot. Das Korn ist eingebracht, der Herbst ist da. Die Dunkelheit kommt heran, Luna ist dabei, ihr Sternentuch zu breiten. Wenn man will, kann man in den Göttergestalten auch eine Abfolge der Temperamente sehen, wobei Aurora das sanguinische, Apoll das cholerische, Diana-Luna - das hat eine lange Tradition - das melancholische Temperament einsteht und schließlich für die Verkörperung der Nacht (Abb. 6), die auch Alter, Winter und damit Saturn in sich fasst, auf das phlegmatische Temperament verwiesen wird - noch im 18. Jahrhundert, etwa bei Daniel Chodowieckis Temperamentendarstellung, ist der Phlegmatiker kurz vorm Einschlafen. ${ }^{8}$ Der Bärtige hier hat die Augen geschlossen, Sterne und Nachtgetier umgeben ihn. In der Landschaft findet ein spätwinterlicher

8 Daniel Chodowiecki. Bürgerliches Leben im 18. Jahrhundert, Ausst.-Kat. Städelsches Kunstinstitut und Städtische Galerie. Frankfurt a. M. 1978, Kat.-Nr. 10, Abb. S. 13. 
Fastnachtsumzug in Kostümen unter Fackelschein statt, doch, worauf besonders die Verse abheben, auch Krankheit und Tod nähern sich. In anderen graphischen Serien, etwa von Tobias Verhaecht, gehören zum Dunkel der Nacht auch die menschlichen Abgründe, Mord und Raub, als wären sie zu verbergen. ${ }^{9}$

Man könnte die Verschränkung der verschiedenen Zeitenvorstellungen noch weiter verfolgen, die christliche Fundierung hervorkehren, doch hier soll es genügen, nicht ohne festgestellt zu haben, dass Tagesund Jahreszeitenfolgen grundsätzlich auf dem Lande spielen, die Einbindung in den Naturzyklus einerseits und in eschatologische Vorstellungen andererseits zum Programm gehören. Die beiden Zeitmodelle sollen sich durchdringen. Das wird in der Romantik, bei Philipp Otto Runge oder Caspar David Friedrich wieder aufgegriffen werden. Doch in der Zwischenzeit greift ein säkulares, aufklärerisches Zeitmodell durch radikale Verzeitlichung, und ihm müssen wir uns jetzt zuwenden, in zwei Schritten. Erst wird Hogarths Serie vorgestellt und an der Tradition gemessen und dann soll ihr Verständnishorizont erhellt werden. Schon jetzt ist zu sagen, dass er als Voraussetzung die Erfahrung der Großstadt London hat und dass er sein primäres Vorbild in der augusteischen Moral gefunden hat, im Umkreis der Moralischen Wochenschriften "Tatler", "Spectator" und "Guardian" um 1710 und der satirischen Dichter wie Swift und Gay und dabei die Tradition der sog. Cries, der Kaufrufe der einzelnen Berufsstände in graphischen Serien seit dem 16. Jahrhundert und der Städtebeschreibungen adaptiert, die in England etwa durch Edward "Ned « Ward kurz vor dem Erscheinen der Moralischen Wochenschriften in Umlauf gebracht werden, zum Beispiel im monatlichen Journal des "London Spy « von 1698-1700. ${ }^{10}$ Alle diese Unternehmungen propagieren einen unmittelbaren Gegenwartsbezug in Kunst, Dichtung und Moral und wenden sich mit Nachdruck gegen überkommene Verklärungen in Mythos, Geschichte und Dichtung.

Hogarths Morgen (s. Abb. 1) aus der Tageszeitenserie, die, wie so oft bei Hogarthschen Bildern, zuerst, und zwar 1736, in gemalter Fassung vorlag und im graphischen Zyklus von 1738 auf Grund der Umkehrung

9 Shesgreen 1983 (wie Anm. 1), 44-51, Abb. 9-12, die Nacht Abb. 12.

10 Christine Riding: Street Life. In: Hogarth, hg. von Mark Hallett und Christine Riding, Ausst.-Kat. Tate Britain. London 2006, 119-121 und Kat.Nr. 70. 
durch den Druckvorgang seitenverkehrt erscheint, ${ }^{11}$ Hogarths Morgen die farbige Fassung macht es unhintergehbar, auf den Dächern liegt Schnee - ist im Winter angesiedelt, es ist bitter kalt. Schon der Beginn der Serie kann also deutlich machen, dass die Verhältnisse auf den Kopf gestellt sind. Und genauer Betrachtung kann nicht entgehen, dass der Mittag auf den Herbst, der Abend auf den Sommer und die Nacht paradoxerweise auf den Frühling verweist. Am Morgen prallen zwei Sphären aufeinander. Während sich die von der Nacht Übriggebliebenen vor Tom King's Coffee House, in dem eine Prügelei ausgebrochen ist - eine Perücke fliegt in hohem Bogen aus der Türe -, so sie der müßiggängerischen Jeunesse dorée angehören, körperlichen Genüssen hingeben, versuchen andererseits ältere Frauen aus niederer Gesellschaftsschicht sich am Feuer zu wärmen oder um Almosen zu betteln, was eine, nach Auskunft des Gemäldes gelb gewandete ältere Jungfer mit roter Schürze entweder geflissentlich übersieht oder gar nicht wahrnehmen kann, da sie ob der sich vor ihren Augen vollziehenden Unzucht wie geblendet ist. Sie strebt der Kirche zu, es ist Schlag sieben Uhr in der Früh. Bei der Kirche handelt es sich um St. Paul's Church in Covent Garden, sehr unkirchlich palladianisch von Inigo Jones entworfen. Tom King's Coffee House befand sich ursprünglich an der gegenüberliegenden Seite des Platzes, Hogarth hat es vor die Front der Kirche verlegt, um die Gegensätze aufeinander prallen zu lassen: eigentliches, wenn auch ordinäres Leben und vertrocknete Wohlanständigkeit, die sich der Wirklichkeit entzieht. Der Jungfer folgt ihr verfrorener Bursche mit dem Gebetbuch, er läge entschieden lieber noch im Bett. Die Hintergrundszenen und Häuser lassen sich zwar auch identifizieren, brauchen uns aber nicht zu interessieren. Wichtiger ist zweierlei: dass schon zeitgenössisch, und zwar von Henry Fielding in "Tom Jones ", ${ }^{12}$ die Jungfer als passende, parodistische Paraphrase auf Aurora gesehen wurde, ihr gelbes Gewand mit roter Schürze markiert einen besonderen Sonnenaufgang. Das übrige Personal könnte nach ihrem Auftauchen die Uhr stellen, jeden Morgen dürfte sie zur gleichen Zeit zur Kirche streben. Das ist das eine, das andere wird durch die roten Rüben und die Zwiebeln im Vordergrund gegenüber dem Feuer markiert. Feuer und Winterfrüchte sind traditionelle Attribute der kalten

11 Zur Gemäldeserie mit farbigen Abbildungen: ebd., Kat. Nr. 67 f., Abb. S. 131-134.

12 Henry Fielding: Die Geschichte des Tom Jones, eines Findlings. In: Henry Fielding: Sämtliche Romane, hg. von Norbert Miller, Bd. 2. München $1966,50 \mathrm{f}$. 
Jahreszeit, etwa auf Gemälden von Teniers oder van Bloemen. ${ }^{13}$ Hogarth wusste also, was er tat. Allein sei noch darauf hingewiesen, dass hinter dem Burschen der Jungfer zwei Kinder zu erkennen sind mit Schultaschen auf dem Rücken. Statt, wie es sich für den Morgen gehören würde, zur Schule zu streben, finden sie es auf dem Markt viel interessanter. Auch sie also konterkarieren die klassische Moral.

Die nächste Szene, Noon (s. Abb. 2), hat wiederum eine deutliche Zweiteilung, und auch hier ergreift Hogarth Partei für's Leben der unterklassigen städtischen Bevölkerung und macht sich über die gepflegten Manieren der gehobenen Schichten lustig. Die Bereiche sind deutlich durch eine schmale Abwasserrinne getrennt, über der im Vordergrund eine tote Katze liegt, die wie ein Warnzeichen das Überschreiten der Grenze verhindert. Verstärkt wird die Barriere auch durch den Holzpfeiler, gekrönt durch einen Krug, die Rinne führt genau auf ihn zu. Auf der einen Seite geht's um die leibliche Nahrung, auf der anderen um die geistige, doch die Bigotterie ihrer Anhänger wird hervorgekehrt. Der Anspielungen sind auch hier viele. Die Kirche und damit die Örtlichkeit lassen sich wieder identifizieren, eine bestimmte Londoner Gegend in Soho ist gemeint mit St. Giles-in-the-Fields in der Mitte. Die fromme Gemeinde soll aus französischen hugenottischen Refugés gebildet sein. Sie sind aufgeputzt, heftig geschminkt und manieriert. Das Klischee vom französischen Tanzmeister, das Hogarth, der Franzosenhasser, mehrfach bemüht, ist auch hier zitiert, mit Natur hat diese Seite nichts zu tun. Umso mehr die andere. Wieder wird heftig karessiert, was eine Kettenreaktion auslöst. Die typische Hogarthsche Schöne, der sehr direkt an die Brust gegangen wird, achtet nicht mehr auf ihre Pastetenplatte, das heiße Öl läuft herab, trifft den Arm ihres begleitenden Knaben, der seinerseits vor Schmerz seine Schüssel abrupt auf einem Pfahl abgesetzt hat, die Platte zerbricht, das Essen findet sich nun weitgehend am Boden, was ein kleines Mädchen nutzt, um die herabgefallenen Brocken zu verschlingen. Hogarth lässt andererseits seine englische Cook-maid die klassische Venus übertreffen, das mag uns darauf hinweisen, dass wir hier eine Londoner Venus mit ihrem besonderen Cupido sehen sollen, was insofern Sinn macht, als neben Apoll auch Venus in den Tageszeitenserien für den Mittag einstehen kann. Die Londoner Venus, schon was die identische Kopfneigung und die roten Wangen angeht, ist der

13 Zahlreiche Beispiele der niederländischen Tradition im "Niederländischen Index" (D.I.A.L.) unter den Nummern 23 D 40-44, 23 K 11-43, 23 R 11-20. 
aufgetakelten Französin auf der anderen Seite gegenübergestellt - hier natürliche Röte, dort Schminke, die nicht Jugend und Schönheit ersetzen kann. Dass der Cupido eines der weinenden Kinder aus Poussins Sabinerinnenraub zitiert, ${ }^{14}$ mag zusätzlich darauf verweisen, dass es Hogarth um angemessene Zuordnungen geht. Das eigentlich Klassische gehört nicht auf die Adelsseite, sondern ins wahre Leben. Die Forschung kann mit dem Drachen, der an der Dachrinne der französischen Kirche gestrandet ist, nichts Rechtes anfangen, spekuliert in diese und jene Richtung seit Hogarths Tagen. Die Lösung scheint einfach: Drachensteigen findet im Herbst statt, so hätten wir hier den Jahreszeitenverweis.

Die dritte Szene, der Abend (s. Abb. 3), dagegen steht eindeutig für den Sommer. Der Tag ist heiß gewesen, die Londoner haben sich an den nördlichen Rand der Stadt begeben, zu Sadler's Well am neuen Kanal, also zum einschlägigen Vergnügungstheater in die vermeintliche Sommerfrische. Die Familie im Vordergrund, reich mit Kindern gesegnet, ist auf dem Heimweg, die energische, grimmige Matrone, schon wieder hoch schwanger, hat einen knallroten Kopf, fächelt sich Luft zu und blendet damit ihr bedauernswertes Ehegespons aus, das eines der Kinder zu schleppen hat. Das Männlein trägt's mit Duldermiene, er steht unter dem Pantoffel. Die Kuh hinter ihm setzt ihm Hörner auf, mit dem werdenden Kind scheint ihn seine Frau auch noch betrogen zu haben. Die Darstellung auf ihrem Fächer hat zu Spekulationen Anlass gegeben. Eine Forschungsmehrheit ist der Meinung, Diana und Aktäon seien dargestellt, das scheint als Anspielung zu passen. ${ }^{15}$ Diese Diana, die Mondgöttin, ist alles andere als keusch gewesen, dem Ovidschen Aktäon setzt die Göttin ein Hirschgeweih auf, um ihn in einen Hirschen zu verwandeln, über den seine Hunde, die Verkörperungen der Lüste, herfallen werden. Der Familienhund dagegen trottet erschöpft voran. So schön das Gedankenspiel auch ist, das Fächerbild lässt diese Identifikation nicht zu. Sowohl im Gemälde, wie in der Graphik kann man vorm Original eindeutig identifizieren, um welche Szene es sich handelt: Um Venus, der

14 Nicolas Poussin: Sabinerinnenraub, 1634, The Metropolitan Museum of Art, New York; zur bis in die Antike zurückreichenden Tradition des weinenden Knaben: Werner Busch: Die Entblößung des Mythos als Freilegung der Natur. Rembrandts Ganymed jenseits der Ikonographie. In: Rembrandt van Rijn. Die Entführung des Ganymed, hg. von Uta Neidhardt und Thomas Ketelsen, Ausst.-Kat. Kunstsammlungen Dresden, Gemäldegalerie Alte Meister, Kupferstich-Kabinett. Dresden 2006, 35.

15 So etwa Ausst.-Kat. Hogarth (wie Anm. 10), 135. 
es nicht gelingt, den zur Jagd drängenden Adonis zurückzuhalten. Alle ikonographischen Details sprechen dafür, man braucht nur mit Tizians berühmter Version des Themas zu vergleichen. ${ }^{16}$ Adonis strebt lanzenbewaffnet von Venus fort, wendet sich noch einmal zurück, sie versucht ihn zu halten, wird begleitet von ihrem geflügelten Amor. Auch diese Geschichte macht in ihrer ironischen Umkehr Sinn. Diese städtische Diana ist eher eine ordinäre Venus, hat ihren Adonis abgelegt und sich anderweitig orientiert. Wie dem auch sei, an Anspielungen fehlt es auch hier nicht. Im Gegensatz aber zu allegorisch-emblematischer Eindeutigkeit eröffnet die Anspielung nur Möglichkeiten, die der Betrachter nach seinem Gusto aufgreifen kann oder nicht. Der Ort, an dem die Szene sich ereignet, ist markiert, doch welche Abgründe sich hinter den Ausflüglern verbergen, darüber können wir nur spekulieren - aber eben das macht den besonderen Reiz aus.

Die vierte Szene, die Nacht (s. Abb. 4), ist auf den Jahrestag genau zu fixieren. Es ist der 29. Mai, der Restauration oder auch Oak Apple Day - und Eichenlaub ist überall angebracht. Das Denkmal im Hintergrund zeigt Charles I., wir befinden uns in Charing Cross. Sein Sohn Charles II. hatte 1660 die Stuart Dynastie wieder hergestellt. Die Feier dieses Tages war in Georgianischen Zeiten verboten, doch die StuartAnhänger konnten sich hinter der unmittelbar vorangehenden Feier zum Geburtstag Georg I., am 28. Mai, verschanzen. Auch hier hat es die Forschung nicht so leicht, die Anspielungen auf die traditionelle Tageszeitenikonographie zu entschlüsseln. Wie könnte auf die Verkörperung der Nacht oder auf Saturn oder in antiker Tradition auf Proserpina verwiesen sein? Letztere wollte die Forschung in der protestierenden Alten in der umgestürzten Kutsche sehen, ersteren im torkelnden Freimaurer im Bildvordergrund. ${ }^{17}$ Zwingend erscheint das nicht. Hogarth, der selber Freimaurer war, dürfte um die Freimaurerikonographie gewusst haben. Die englische Großloge wurde 1717 in London gegründet. Desaguilier wurde 1719 Großmeister der Loge und war verantwortlich für die zentrale Ordnungsschrift der Freimaurer unter dem Titel »Moon«. Die Loge wur-

16 Zur Pose bei Tizian: Jan L. de Jong: Word Processing in the Italian Renaisssance: Action and Reaction with Pen and Brushwork. In: Visual Resources 19, 2003, bes. 263 f. und Werner Busch: Das unklassische Bild. Von Tizian bis Constable und Turner. München 2009, 110 und Abb. 52.

17 Paulson 1989 (wie Anm. 1), 107. 
de als ein Abbild des Kosmos begriffen, regiert von Sonne und Mond. ${ }^{18}$ $\mathrm{Ob}$ dies etwas für unsere Darstellung, in der der Mond durch die Wolken segelt, zu bedeuten hat, bleibe dahin gestellt. Deutlich ist erneut die Gegenüberstellung. Das nächtliche Treiben in der Bordellgegend, in der die Nacht zum Tage gemacht wird von denen, die es sich leisten können, steht im Gegensatz zum Verhalten der ärmlichen Bevölkerung, die verzweifelt unter dem Fenster des Barbiers zu schlafen sucht, wobei der kleine Knabe bereits seine Fackel entfacht. Er ist ein link-boy, der des Nachts den Nachtschwärmern auf dem Heimweg leuchtet.

So bringt das Leben in der Großstadt die Ordnung durcheinander, traditionelle Abläufe zählen nicht mehr, aber auch die Bilder, die vergangenen Weltvorstellungen Ausdruck verliehen, können keine Geltung mehr beanspruchen. Für die neuen Erfahrungen war eine Sprachregelung zu finden, die zugleich Anleitung geben konnte, mit den neuen Anforderungen fertig zu werden. Dies unternahmen Stadtbeschreibungen, die Moralischen Wochenschriften und satirische Dichtungen, die alt und neu aufeinander prallen ließen. Edward "Ned" Ward zielte mit seinen Stadtbeschreibungen auf ein breites Publikum, ihm ging es nicht um Highlights, um historisch Bedeutendes, sondern um die City in ihrer Vielfalt, ihre Eitelkeiten und Laster, er zog durch die Tavernen und Kaffeehäuser, über die Märkte, berichtete von Festen und Festivals, bevorzugte das Alltägliche, hatte Komisches und Abstruses zu berichten, besonders in seiner Monatsschrift "The London Spy « (1698-1700). Mit einem Freund, der sich auskannte, erwanderte er London zu Fuß, warf einen frischen Blick auf Allbekanntes, machte die Londoner mit ihrer eigenen Stadt bekannt, indem er neugierig durch jedes Schlüsselloch schaute, aber auch er ist genervt durch den Dauerlärm auf den Straßen. ${ }^{19}$

Hogarth hat diese Erfahrung in seinem Enraged Musician (Abb. 7) verewigt - aber auch da den Straßenlärm als Ausdruck wirklichen Lebens begriffen und den leidenden Musikmeister, bei dem es sich, wie sich hat nachweisen lassen, um einen italienischen Violinisten aus Händels Opernorchester gehandelt hat, nur lächerlich gemacht, da er einer

18 Zur englischen Freimaurerei: Werner Busch, Materie und Geist. Die Rolle der Kunst bei der Popularisierung des Newtonschen Weltbildes. In: Mehr Licht. Europa um 1770. Die bildende Kunst der Aufklärung, Ausst.-Kat. Städelsches Kunstinstitut und Städtische Galerie, Frankfurt a. $M$. München 1999, 410-414.

19 S. Anm. 10. 


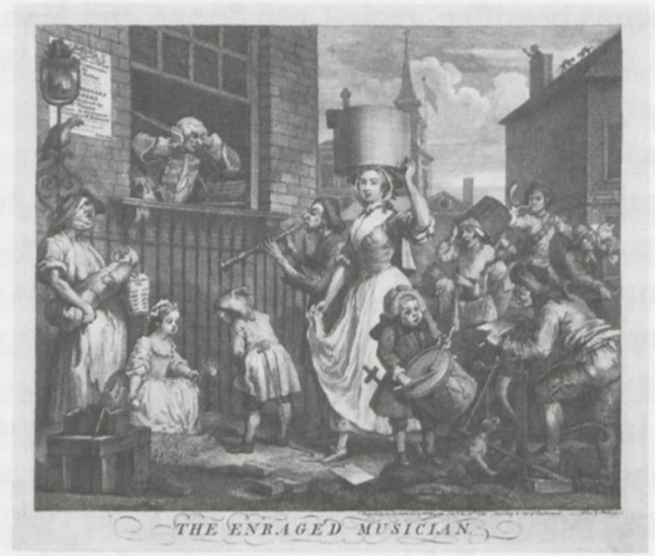

7 William Hogarth: The Enraged Musician, Radierung und Kupferstich, $1741,35,7 \times 41,2 \mathrm{~cm}$

überholten Auffassung von Musik anhängt. ${ }^{20}$ Der Kampf der italienischen Oper, der in den 1730er Jahren zugunsten der nationalsprachlichen volkstümlichen Oper, etwa der "Beggar's Opera" von John Gay, entschieden wird, nahm seinen Ausgang in den »Moralischen Wochenschriften «, in denen auch der Kampf gegen den tradierten Mythos und seine Bilder geführt wurde. ${ }^{21}$ In beiden Fällen hat Joseph Addison im "Spectator " die Feder gespitzt, gegen die Oper bereits in Nr. 5 vom 6. März 1710/11, ${ }^{22}$ gegen den Mythos und für die Zeitgenossenschaft der Dichtung in Nr. 523 vom 30. Oktober 1712. Hier moniert er, dass jede Würdigung eines bedeutenden Mannes sich der Ovidschen Metamorphosen bedient, was er nur noch für überflüssigen Schulkram hält. Jedes Gedicht auf eine vornehme Frau bemühe den Vergleich mit Venus oder Helena, ob er passt oder nicht. Wenn man eine wackre Panegyrik schreibe, dann solle sie alle Farben der Wahrheit tragen, und nichts könne lächerlicher

20 Paulson 1989 (wie Anm. 1), Kat.-Nr. 132, S. 110 f.; David Bindman: Hogarth and his Times, Ausst.-Kat. British Museum. London 1997, Kat.-Nr. 71; Werner Busch: Kakophonie! William Hogarth's The Enraged Musician. In: Freia Hoffmann, Markus Gärtner und Axel Weidenfeld (Hg.): Musik im sozialen Raum. Festschrift für Peter Schleuning zum 70. Geburtstag. München 2011, 58-74.

21 Werner Busch: Händel und der Wandel der Konversation. In: ders.: Englishness. Beiträge zur englischen Kunst des 18. Jahrhunderts von Hogarth bis Romney. Berlin/München 2010, 9-31.

22 The Spectator, Bd. 1, Nr. 5, 6. März 1710/11, London o.J. (um 1790), 21-25. 
sein als ein steter Rekurs auf Jupiter oder Juno. Es gäbe nur einen Ort, an dem es Sinn mache, die heidnischen Götter zu bemühen und das sei in der mock-heroischen Dichtung. Die Anwendung des gesamten klassischen Apparates ("the fabulous machines of the ancients «) auf niedere Gegenstände, mache den Apparat automatisch lächerlich, kehre hervor, wie inadäquat er in der Gegenwart sei. Aber auch in klassischen Dichtungsformen wie der Pastorale sei es durchaus möglich, auf Faune und Satyrn, auf Wald- und Wassernymphen und all die ländlichen Gottheiten zu verzichten. Man müsse der Gattung nur neues Leben einhauchen, die natürliche Schönheit bemühen und die antiquierten Fabeln abergläubischer Mythologie über Bord werfen. Man müsse Realitäten, nicht Fiktionen beschreiben. Und dann kommt es am Ende des Textes von Addison zu einer Ausweitung des Argumentes, dessen Bedeutung für das Folgende gar nicht zu überschätzen ist. Er führt als Beispiel den viel zitierten Neptun an und verbittet sich schlicht auch dessen Erscheinen, "unless it be done in metaphor, simile, or any very short allusion«. Und selbst in diesen Fällen solle man äußerst vorsichtig und zurückhaltend agieren. Gottheiten als bloße Metaphern, als Gleichnis oder kurze Allusion, sind in Grenzen zugelassen, was impliziert, dass sie in Form ausgeführter Allegorie oder in symbolischer Funktion kein Vorkommen mehr haben sollen. Als Sprachornat ja, als Verkörperung von Wahrheiten nein. ${ }^{23}$

Die Wahrheiten spiegeln sich allein in den sozialen Wirklichkeiten, und die sind auf eine präzise Schilderung gegenwärtiger Verhältnisse angewiesen, und auf sie stößt den Betrachter mit Notwendigkeit die Stadt. Um ihr allerdings Herr zu werden, braucht's Anweisung und Moral. Bei der Aneignung der Stadt jedoch in all ihren Facetten kommt es, um es so zu sagen, auch zu einem neuen Zeitmaß. Zwei Beispiele. John Gay, der »Beggar's Opera" 1728 verfasst hat, deren Hauptszene sofort in nicht weniger als sechs Fassungen von Hogarth mit ausgeprägtem Tagesbezug verewigt wurde, ${ }^{24}$ hatte zuvor schon 1716 die Versdichtung "Trivia: Or, the Art of Walking the Streets of London " veröffentlicht. ${ }^{25}$ Man kann das Gedicht eine städtische Gebrauchsanweisung nennen. Nichts wird

23 The Spectator, Bd. 7, Nr. 523, 30. Oktober 1712, London o.J. (um 1790), 198-201, die wörtlichen Zitate 199 und 200.

24 David Bindman und Scott Wilcox (Hg.): „Among the Whores and Thieves«. William Hogarth and »The Beggar's Opera». Yale Center for British Art, New Haven 1997.

25 John Gay: Trivia: Or, The Art of Walking the Streets of London, London 
ausgelassen. Buch 1 beginnt mit der Ausrüstung des Stadtwanderers, der genau das Wetter beobachten soll, um richtig angezogen zu sein. Auf sein Schuhwerk hat er zu achten: »Be thou, for ev'ry Season, justly drest ... ${ }^{26}{ }^{26}$ Die Jahreszeiten haben nur noch ihr Vorkommen in Hinblick auf angemessene Bekleidung. Stundenlang wird das schlechte Londoner Wetter beschrieben, der Dreck auf den Straßen beklagt. Einen Schirm soll man dabei haben - wenn's allzu schlimm wird, sich in einem Laden unterstellen.

Buch 2 beschreibt die Tageswanderungen und beginnt mit dem Morgen. Nicht die Morgenröte wird in ihrem göttlichen Glanz beschworen, vielmehr heißt es: »For Ease and for Dispatch, the morning's best:/ No tides of Passenger the Street molest «, ${ }^{27}$ »Wenn man es bequem haben will und in Eile ist, dann ist der Morgen am besten, keine Flut von Fußgängern belästigt einen auf der Straße.« Ein sehr anderes Morgengefühl wird sichtbar. Man beobachtet, wie die Stadt erwacht, wie die Hausierer sich geschäftig auf den Weg machen, die Läden öffnen, die Wagen anfangen, die Straßen zu bevölkern, Karren durch den Morast gezogen werden, wie schließlich alle Straßen von Lärm und Kaufrufen erfüllt sind. Das führt den Stadtwanderer dazu, die einzelnen Berufssparten anzuführen, derer man auf der Straße ansichtig wird, den Barbier, den Bäcker, den Schornsteinfeger, den Müllkutscher. Der Porter, der Träger, hat sein besonderes Vorkommen, er kennt sich besonders gut aus, ihn soll man nach dem Weg fragen. Dann kommt es zur Addisonschen Nutzanwendung: Entweder gibt es metaphorische Götterverweise - man braucht einen Ariadnefaden im Stadtgewirr - oder der Göttervater Jupiter, der ständig nach neuen Liebschaften Ausschau hält - "great Jove ... of old « heißt es ausdrücklich ${ }^{28}$ - war auch nicht besser als all die Vielen, die ihren irdischen Leidenschaften nachgehen. Ein Vers hebt scheinbar konventionell an: "Now dawns the Morn «, »Nun dämmert der Morgen«, doch der Vers geht weiter »the sturdy Lad awakes $«,{ }^{29} »$ der derbe Bursche wacht auf «. Der Stadtwanderer warnt vor gewissen Straßen, macht sich besonders über Pall-Mall lustig, wobei man wissen muss, dass hier im 18. Jahrhundert die ausgehaltenen gehobenen Mätressen wohnten.

1716. In: John Gay: Poetry and Prose, hg. von Vinton A. Dearing, Bd. 1. Oxford 1974, 134-181.

26 Ebd., Buch 1, 138.

27 Ebd., Buch 2, 143.

28 Ebd., 145.

29 Ebd., 149. 
Das 3. Buch widmet sich der Stadtwanderung bei Nacht. Notwendig wird zur Vorsicht gemahnt, Taschendiebe treiben sich herum, fragwürdige Balladensänger. Man bekommt Anleitung, wie man eine Hure erkennt etc. Auch die Nacht hat ihre Schönheit verloren, vom Mond ist nicht mehr die Rede, allein von Feuern oder den fackelleuchtenden link-boys. Kein erholsamer Schlaf wird versprochen, kein Gemahnen an den Tod wird vorgeführt. ${ }^{30}$ Nun gibt es für manches, das hier aufgerufen wird, durchaus literarische oder bildkünstlerische Vorläufer, und aus ihrem Repertoire bedient sich auch Hogarth. All die Straßenberufe zum Beispiel hatten seit dem späten 16. Jahrhundert in den sogenannten Cris de Paris oder den Cries of London in Serienform ihr Vorkommen. Die typischen Berufskleidungen wurden vorgeführt und mit den jeweiligen Kaufrufen in Versform versehen. Auch diese Serien haben didaktische Funktion, machen mit dem Stadtkosmos vertraut. Auf Hogarths Enraged Musician kann man sie reihenweise ausmachen und aus der Cris-Tradition herleiten. Berühmte Ursprungsserie ist Annibale Carraccis Folge der Berufe in mehr als 80 Blättern, ihr folgt eine populäre Tradition Bologneser Graphik bis ins 18. Jahrhundert, von der man weiß, dass sie nicht ohne Einfluss auf Hogarth gewesen ist. ${ }^{31}$

Wichtiger noch zum Verständnis der besonderen Form von Hogarths Tageszeitenserie ist jedoch Swifts Satire auf den Morgen, die Richard Steele im "Tatler Nr. 9" vom 28. April 1709 herausgegeben hat. ${ }^{32}$ Swift und Hogarth haben sich wechselseitig besonders geschätzt, wobei, schon von der Chronologie her, Swift mehr der Gebende und Hogarth der Nehmende war. Die Swiftsche Morgenbeschreibung dürfte auch deshalb von besonderem Einfluss auf Hogarth gewesen sein, weil Swift die traditionelle Morgenschilderung auf den Kopf stellt, einen neuen gänzlich städtischen Zeitenbegriff entwickelt. Das Tatler-Stück beginnt damit, dass beschrieben wird, was die Autoren Swift und Steele am Abend gemacht haben. Sie waren im Theater, haben die Komödie "The old Bachelor"

30 Ebd., Buch 3, 160-170.

31 Bindman 1997 (wie Anm. 19), 36-40 leitet die Hogarthsche Stadtauffassung von Juvenals dritter Satire her; zur Cries-Tradition: Ausst.-Kat. Hogarth 2006 (wie Anm. 10), Kat.-Nr. 60-63, S. 124-126; Kat.-Nr. 72, S. 138. $\mathrm{Zu}$ den Arti von Annibale Carracci: Le arti di Bologna di Annibale Carracci, hg. von A. Marabottini. Rom 1979.

32 Angus Ross (Hg.): Selections from The Tatler and The Spectator of Steele and Addison. Harmondsworth 1982, The Tatler Nr. 9, 28. April 1709 (A Description of the Morning), Steele with Swift, 76-78. 
gesehen, ein Stück von William Congreve, in dem ein alter Debauché zur Ordnung gerufen wird. ${ }^{33}$ Eine derartige, wie man es genannt hat, »comedy of manners « mache durchaus Sinn, doch heutzutage sei die Stadt voll mit »Easie Writers", die gänzlich abwegiges Zeug verfassen würden. ${ }^{34}$ Ein Verwandter von ihm dagegen, schreibt Steele in seiner Einleitung, und gemeint ist offensichtlich Swift, kämpfe gegen den Strom der Absurditäten an und beschreibe auf gänzlich neue Weise Dinge »exactly as they happen: He never forms Fields, or Nymphs, or Groves, where they are not, but makes the Incidents just as they really appear « ${ }^{35}$ Das argumentiert mit Nachdruck gegen die verklärende Pastorale mit ihrem »locus amoenus", mit sich darin in verborgenen Grotten tummelnden ländlichen Naturgottheiten nackt beim Bade. Er habe, schreibt Steele, diesem Verwandten, gemeint ist im Geiste, ein Manuskript mit einer gedichteten Beschreibung eines Morgens gestohlen, »but of the Morning in Town « und zwar aus der Stadtgegend, wo sein Verwandter zur Zeit wohne, und er scheue sich nicht, dieses Gedicht abzudrucken, als ein Beispiel für eine neue Dichtungsform. ${ }^{36} \mathrm{Da}$ der Autor jedoch fürchte, von anderen nachgeahmt zu werden, vermerkt Steele am Ende, habe er etwa dessen Beschreibung des Abends nicht abgedruckt. In ihr würden Dirnen beginnen, in den Straßen und Gängen um das Schauspielhaus herumzuschlendern - gemeint ist, um Ausschau nach Kunden zu halten. Auch den Mittag habe er ausgelassen, eine Zeit, in der die feinen Damen und die großartigen Stutzer gähnend aus ihren Betten stiegen und in Pall-Mall aus dem Fenster schauten. ${ }^{37}$ Also kein Lustwandeln im Park unter der Protektion der Venus, wie in den überlieferten Tageszeitenserien, sondern die Realität der Großstadt, wo die Mätressen sich aushalten lassen, keine unschuldige Lustbarkeit am Abend nach des Tages Arbeit, sondern Prostitution. Steeles Andeutung der Tendenz der nicht zum Abdruck gebrachten Teile des Tageszeitengedichts macht deutlich, dass er genau das Gegenteil von dem erreichen will, was der Autor vermeintlich befürchtet: Er wünscht sich Nachfolge für diesen literarischen Typus neuer Wirklichkeitspoesie.

Der Morgen hebt an, wie auch Gay angehoben hat. Am frühen Morgen sind die Straßen noch leer, erste Lohnkutschen tauchen auf, doch

33 Ebd., 76.

34 Ebd., 77.

35 Ebd.

36 Ebd.

37 Ebd., 78. 
dann wird's gleich direkt. Eine Betty schleicht sich aus dem Bett ihres Meisters, zerwühlt in ihrer Kammer ihr eigenes Bett ein wenig, um zu vertuschen, wo sie die Nacht zugebracht hat. Ein schlampiger Lehrling treibt sich vor seines Meisters Türe herum, Moll dagegen schrubbt den Eingang und die Stufen. Die tiefen Spuren auf der Straße am Rinnstein werden eingeebnet, der Kohlenmann preist mit dunkler Stimme seine Ware an, übertönt von schrillen Aufforderungen des Schornsteinfegers die Tradition der Cries ist allgegenwärtig -, vorm Tor seiner Lordschaft versammeln sich seine Gläubiger, der Lärm nimmt zu. Als Krönung des morgendlichen Durcheinanders erwartet der Gefängniswärter, wie es heißt, seine Herde, die er pünktlich zur Nacht zu Raubzügen herausgelassen hat, damit sie die Gefängnisgebühr bezahlen kann. ${ }^{38}$ Das entsprach in der Tat einer sozialen Realität. Auf lange Sicht eingekerkert für die banalsten Vergehen mussten die Gefangenen für alles zahlen; wehe, sie konnten es nicht. Die Gefängnisse waren vom Staat vermietet an private Betreiber, die die Gefangenen ausnahmen, wo es nur ging. Konnten sie nicht zahlen, wurden sie auf Streifzüge geschickt. Die Gebrüder Fielding und vor allem der Gefängnisreformer John Howard versuchten diese haltlosen Zustände zu ändern, Erfolge stellten sich erst in der zweiten Jahrhunderthälfte ein. ${ }^{39}$ Swifts Diebesherde, die am Morgen heimkehrt, konterkariert die traditionelle Morgenikonographie mit der ausziehenden Herde. Das Swiftsche Schlussbild bilden Schulkinder mit ihren Schulmappen, die nicht den direkten Weg zur Schule einschlagen von hier dürften sie ihren Weg in Hogarths Darstellung des Morgens (s. Abb. 1) gefunden haben. ${ }^{40}$

Was lässt sich aus alledem schließen? Sicher wäre es monokausal argumentiert, wollte man allein die entstehende Großstadt mit ihren besonderen Strukturen für den Wandel in der Auffassung der Zeiten verantwortlich machen. Um es verkürzt zu sagen, auch Newton und die neuen physikalischen Erkenntnisse haben ihren Anteil. Doch die Stadt scheint dazu zu zwingen, sich der Gegenwärtigkeit zu stellen, Anzeichen, Details ernst zu nehmen. Bekommen jedoch Wirklichkeitspartikel auch in der Kunst ihr Erscheinungsrecht als Erfahrungstatsachen, die erst

\section{Ebd., 77.}

39 Werner Busch: Romneys Howard. Revolution und Abstraktion. In: Städel-Jahrbuch, N. F. 16, 1997, 289-332; wieder abgedruckt in: Busch 2010 (wie Anm. 21), 171-221.

40 Ross 1982 (wie Anm. 32), 77: "The watchful Bailiffs take their silent Stands / And School-boys lag with Satchels in their Hands«. 
Erkenntnisprozesse zulassen, dann kommen die klassischen Idealisierungsformen an ihr Ende, die gefordert hatten, keine "particularities" oder "minuteness « zuzulassen, sondern "generality « anzustreben. ${ }^{41} \mathrm{Mit}$ Verklärung ist der Stadt nicht beizukommen, nur aus dem Festhalten individueller Momente ergibt sich ihr Bild. Der individuelle Moment mit all seiner Begrenztheit tritt an die Stelle aller überzeitlichen Vorstellungen. Selbst die Romantik mit ihren Remythologisierungstendenzen kann nicht mehr wie Paul Fleming dichten: "Ach daß doch jene Zeit, die ohne Zeit ist, käme / Und uns aus dieser Zeit in ihre Zeiten nähme " ${ }^{42}$ sondern nur mit Hölderlin zum Morgen schreiben: »segne mir lieber dann / Mein sterblich Tun und heitere wieder / Gütiger! heute den stillen Pfad mir «. ${ }^{43}$ Gottes Schutz wird zwar berufen, doch nur für den gegenwärtigen Moment. Alles andere entzieht sich der Vorstellbarkeit, die Psyche steht dem entgegen.

ABBILDUNGSNACHWEISE

1-7 Archiv des Verfassers.

41 Werner Busch: Zu einer Theorie des Details. Diderot und Richardson, Greuze und Chodowiecki. In: Jenseits der Grenzen. Französische und deutsche Kunst von Ancien Régime bis zur Gegenwart, Thomas W. Gaehtgens zum 60. Geburtstag, Bd.1: Inszenierung der Dynastien, hg. von Uwe Fleckner, Martin Schieder und Michael F. Zimmermann. Köln 2000, 302-317.

42 Zitiert bei Conrady 1992 (wie Anm. 6), 31.

43 Zitiert ebd., 211 (»Des Morgens«), letzte Strophe. 